\title{
Investigating The Status of Quality of Life in Freight Truck Drivers of The City of Kermanshah and Its Influencing Factors
}

Mahnaz Solhi, Professor. Occupational Health Research Center, School of Health, Iran University of Medical Sciences, Tehran, Iran.

Seyed Fahim Irandoost, PhD in Health Education and Health Promotion, Department of Public Health, School of Health, Urmia University of Medical Sciences, Urmia, Iran.

(1) Mitra Abolfathi, ( ${ }^{*}$ Corresponding author), $\mathrm{PhD}$ Candidate in Health Education and Health Promotion, Iran University of Medical Sciences, Tehran, Iran. mabolfathi74@yahoo.com

\section{Abstract}

Background and aims: The quality of life means the individual's interpretation of their place of residence considering the culture and value system of the community, which is influenced by the goals, expectations and standards' sought. There are several factors in driving a car that affects the drivers' quality of life. The purpose of this study was to determine the quality of life of truck drivers of the city of Kermanshah in 2015, and determine its relationship with personal characteristics.

Methods: In this cross-sectional descriptive-analytic study, 258 drivers were randomly selected. The data collection tool was a short form of quality of life questionnaire (SF12). Demographic data was also collected in the profile form. The data were entered into SPSS-20 and analyzed by using descriptive statistical indices and inferential statistics.

Results: The mean and standard deviation of the quality life was $38.5 \% \pm 4.3$. Out of all participants, $66.7 \%$ in the research had a good quality of life. Among the dimensions of the quality of life, the lowest mean score was related to social health $(19.5 \pm 2.7)$, and the highest score was related to mental health $(21.1 \pm 3.4)$. There was a significant difference between the mean score of quality of life, in terms of marital status $(\mathrm{P}=0.042)$, age $(\mathrm{P}=032)$, physical activity $(\mathrm{P}=0.002)$ and history of gastrointestinal diseases $(\mathrm{P}=0.013)$.

Conclusion: The status of quality of life was in a good condition in more than half of the surveyed truck drivers. Elseways, about 33\% of these drivers had a modest or inferior quality of life. Planning for promotion interventions is recommended to improve the quality of life of these drivers.

Conflicts of interest: None

Funding: None

\section{Keywords}

Quality of life

Truck drivers

Physical health

Mental health

Social health

Environmental health

Received: 2018/05/09

Accepted : 2019/08/12 


\section{INTRODUCTION}

Today, transportation has an important and underlying role in the economic growth of the society. Approximately, half a million people in Iran have chosen driving career as their primary profession and they have a key role in the transportation of freight and passengers in the country. One of the most important methods of freight transportation in Iran, is the road transportation by freight trucks on roads. In the driving career, similar to other careers, there are several risk factors that may threaten the health of the drivers, due to being exposed to detrimental career factors. Road accidents are one of the major causes of death and disability in the world. In the developed countries, 1 to 2.5 individuals die for every 10000 vehicles, and 3 to 15 individuals die in traffic accidents. The index is reported to be 29 in Iran (1).

Given the definition of health and well-being by the WHO, based on wide dimensions of full physical, mental and social well-being and not just lack of disease, nowadays, health measurement and evaluation of health interventions, considers others human values such as the quality of life besides calculating mortality, frequency and severity of illnesses (2). The group of the quality of life of the WHO, defines quality of life as: the individual's perception of their position in life in the context of the culture and value systems, in which they live and in relation to their goals, expectation, and standards (3). One of the concerns of employees, is the dangers of the workplace, which may affect their quality of life. Driving, as one of the most difficult and dangerous careers, has rates of injury, death and work absence higher than other occupations. Drivers, in addition to job stress, face numerous career detrimental factors such as working in poor weather conditions, irregular work shifts, excessive overload, carrying heavy loads, excessive work duration and short rest time, shaking and other factors affective on the incidence of musculoskeletal disorders, as well as factors affecting the weakness of the immune system, which makes a person susceptible to work-related diseases (4). One of the major challenges and most common causes of the freight truck drivers' accident incidence, is insomnia; being sleepy while driving, causes over 100000 accidents a year, resulting in 40000 injuries and 1550 deaths (5).

Considering the importance of their health and quality of life, in how they serve their family and perform career duties, the aim of this study was to determine the status of the quality of life of the freight trucks' drivers, in the city of Kermanshah in 2015, and its related factors.

\section{METHODS AND MATERIALS}

This descriptive-analytical type cross-sectional study, was conducted on 258 subjects. The study population, included all the freight truck drivers in Kermanshah city, with at least three years of driving experience (about 750 people). Using the Morgan table, for a population of 750 drivers, a sample of 256 people was selected, where practically 258 drivers participated in the research. The available sampling method, was non-probabilistic. The inclusion criteria had at least three years of experience as a freight truck driver in the Kermanshah city, and signed an informed consents to participate in the study. Dissatisfaction to continue the participation, was also determined as the exclusion criteria. The site of interview with the drivers, was the sites of loading, accommodation and rest areas, and in some cases, their habitat. The data collection tool was the standard questionnaire of short form of quality of life (SF12), which had 12 questions, and included the 4 dimensions of physical, psychological, social relationship and environmental health (2). The scale used for classifying the scores obtained from this questionnaire, is that getting a score of 12 means a poor quality of life, a score of 25 means a moderate quality of life, and a score of $37-48$ equals a good quality of life. The validity and reliability of this questionnaire, were assessed in the study by Nejat et al.: the physical health dimension $(0.70)$, mental health dimension (0.73), social relationships dimension (0.55) and environmental health dimension (0.84) (2). The questionnaire was completed by a trained expert; and by the questionnaire along with interviewing the drivers, so to reduce the likelihood of responding errors. The demographical information of the drivers, was also collected in the profile form. In order to observe the ethical principles, after obtaining ethical approval, while assuring the study subjects that their personal information would be kept confidential and that they're allowed to quit the session, the informed consent was obtained. Data were entered into the SPSS software version 20, and were described using statistical indices of number, percentage, mean and standard deviation. Given that the distribution of the quality of life score, physical health and psychological health, had an abnormal distribution $(\mathrm{P}<0.05)$, to investigate the relationship between the quality of life and its connection with the demographic variables, the nonparametric MannWhitney test was used.

\section{RESULTS}

About $82 \%$ of the studied drivers were married, and about $53 \%$ had less than a high school diploma. Over $90 \%$ of them were non-smokers. The standard deviation and mean age of the drivers, was $35.6 \pm 7.05$ years; and the mean work experience was 10 years with the standard deviation of 6 years. The mean hours of work per day was 10.2 , with a standard deviation of 1.7. Mean and standard deviation of total quality of life, was $38.5 \pm 4.8$. These results were $17.3 \pm 1.9$, 
Investigating The Status of Quality of Life...

Table 1. Mean and standard deviation of quantitative variables

\begin{tabular}{ccccc}
\hline Variables & Mean & SD & Minimum & Maximum \\
\hline Quality of life (Total) & 38.5 & 4.8 & 22 & 48 \\
Physical health & 17.3 & 1.9 & 11 & 20 \\
Mental health & 21.1 & 3.4 & 10 & 28 \\
Social health & 19.5 & 2.7 & 12 & 36 \\
Environmental health & 20.7 & 4.3 & 15 & 59 \\
Age & 35.6 & 7.05 & 23 & 35 \\
History of work & 9.9 & 5.9 & 1 & 18 \\
Hours of work & 10.2 & 1.7 & 4 & \\
\hline
\end{tabular}

Table 2. Distribution of the quality of life among participants

\begin{tabular}{cccc}
\hline Variable & Categories & Frequency & Percentage \\
\hline \multirow{3}{*}{ Quality of life } & Good & 172 & 66.7 \\
& Moderate & 84 & 32.6 \\
& Poor & 2 & 0.8 \\
\hline
\end{tabular}

for the physical health dimension and $21.1 \pm 3.4$, for the mental health dimension (Table 1). $66.7 \%$ of the research participants, had a good quality of life. The quality of life of $32.6 \%$ of the freight truck drivers was moderate, and only $0.8 \%$ of them had a poor quality of life (Table 2).

Using the Mann-Whitney test, a statistically significant difference was observed between the mean score of marital quality of life $(\mathrm{P}=0.042)$, age $(\mathrm{P}=0.032)$, exercise activity $(\mathrm{P}=0.002)$ and gastrointestinal disease history of the individuals $(\mathrm{P}=0.013)$. There was a statistically significant difference between the mean score of the physical health on the dimensions of quality of life, in terms of the variable of working hours $(\mathrm{P}=0.03)$. There was also a statistically significant difference between the mean score of physical health and quality of life, in terms of physical activity $(\mathrm{P}=0.001)$. There was a statistically significant difference between the mean score of quality of life, according to the individuals' history of gastrointestinal disease $(\mathrm{P}=0.007)$. The results of the Mann-Whitney test showed that, there is a statistically significant difference between the mean score of mental health in married and single individuals $(\mathrm{P}=0.028)$. There was a significant difference between the mean score of mental health in diffrent age groups $(\mathrm{P}=0.036)$. There was a statistically significant difference between the mean score of mental health, in individuals with different physical activity $(\mathrm{P}=0.028)$. There was a statistically significant difference between the mean score of mental health, in those with a history of gastrointestinal disease and without a history of the disease $(\mathrm{P}=0.042)$. The Mann-Whitney test showed a statistically significant difference between the mean score of social relationships of single and married individuals $(\mathrm{P}=0.002)$. By comparing the mean score of the social relationships dimension in individuals with different educations, a statistically significant difference was obtained $(\mathrm{P}=0.014)$. By comparing the mean score of the social relationships dimension in individuals with physical activity and those without the activity, a statistically significant difference was obtained $(\mathrm{P}=0.033)$. Also, by comparing the mean score of social relationships dimension in individuals with a history of gastrointestinal disease and those without the disease, a statistically significant difference was obtained $(\mathrm{P}=0.015)$. A statistically significant difference was observed between the mean score of environmental health, in people with different levels of education $(\mathrm{P}=0.001)$. There was also a statistically significant difference between the mean score of environmental health in people with different work experience $(\mathrm{P}=0.012)$.

\section{CONCLUSION}

This study showed that the status of quality of life of over half of Kermanshah's freight truck drivers is good, but about $33 \%$ of them have moderate or poor quality of life, and through adopting new policies and performing improvemental interventions in this regard, their quality of life can be raised. It is recommended that improvemental interventions should be designed to improve the quality of life of these drivers, given their marital status, age, physical activity, and gastrointestinal history. Self-reporting and conducting a study on the drivers of one city, was one of the limitations of this study. It is recommended to conduct a qualitative study, by interviewing drivers 
Solhi M, et al.

and conducting a study on truck drivers in other cities.

\section{ACKNOWLEDGMENT}

This research was supported by the Occupational Health Research Center of Iran University of Medical Sciences: (Grant No. 94-02-13-25854). The study was provided ethical approval by the Iran University of Medical Sciences (IR.IUMS.REC. 94-
02-13-25854). The funders, however, played no role in designing the study, collecting and analyzing data, manuscript preparation, and the decision to publish the manuscript. All participants in this study are appreciated.

\section{CONFLICTS OF INTEREST}

The authors declare that there are no conflicts of interest regarding the publication of this manuscript.

How to cite this article:

Mahnaz Solhi, Seyed Fahim Irandoost, Mitra Abolfathi. Investigating The Status of Quality of Life in Freight Truck Drivers of The City of Kermanshah and Its Influencing Factors. Iran Occupational Health. 2021 (01 Aug);18:15.

*This work is published under CC BY-NC 4.0 licence 


\section{بررسى وضعيت كيفيت زندَى رانندكان كاميونهاى باربرى شهر كرمانشاه و عوامل موثر بر آن}

مهيداز صلحى: يروفسور، مركز تحقيقات سلامت كار، دانشكاه علوم يزشكى ايران، تهران، ايران.

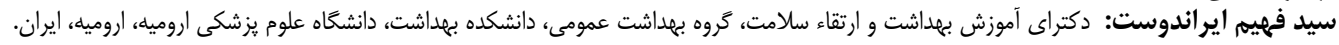

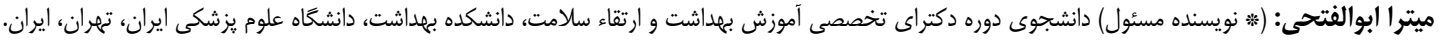
mabolfathi74@yahoo.com

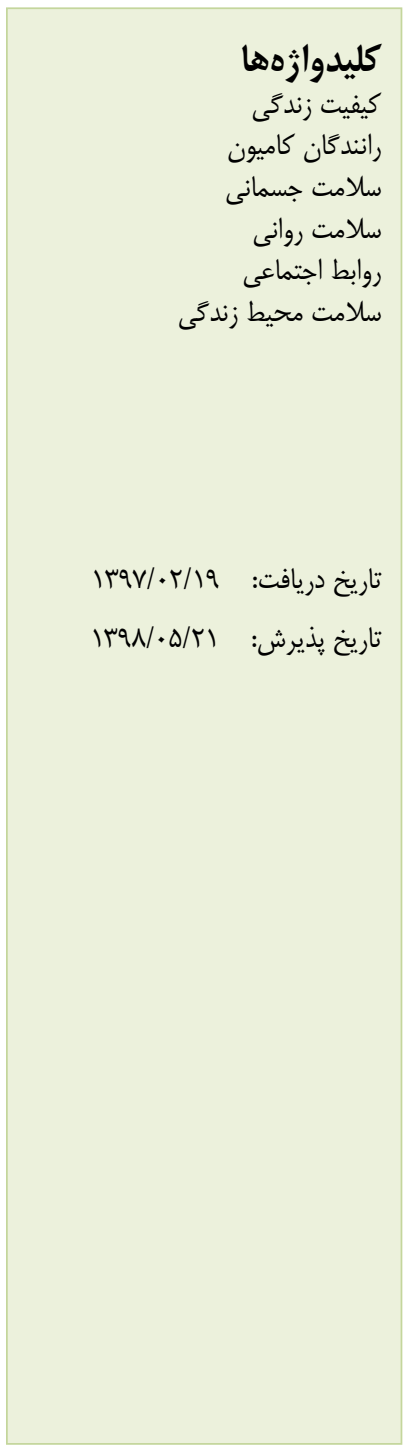

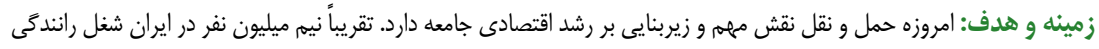

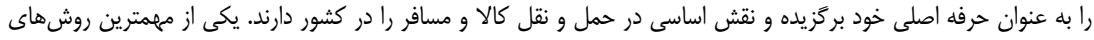

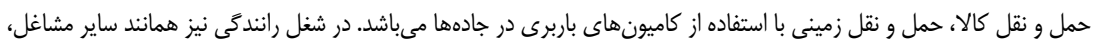

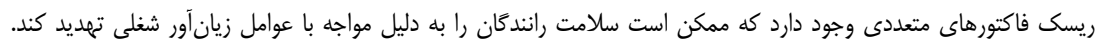

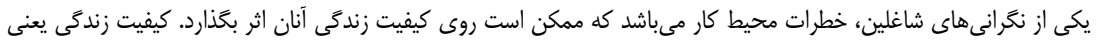

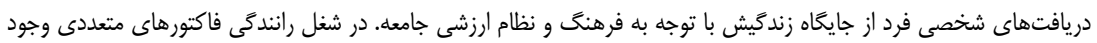

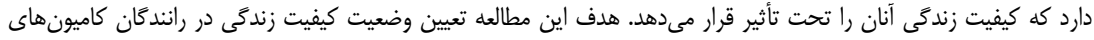

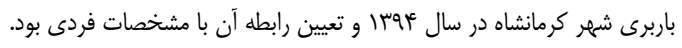

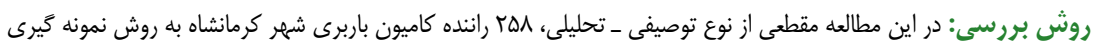

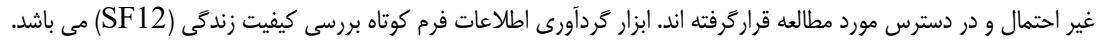

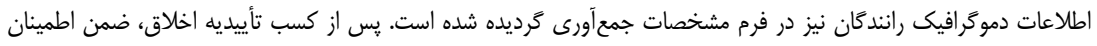

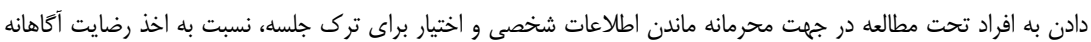

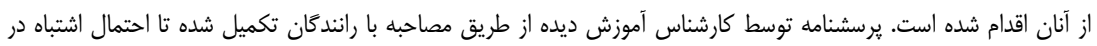

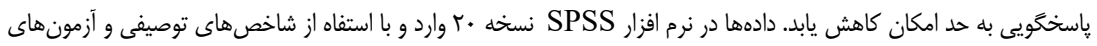
تحليلى تجزيه و تحليل شد.

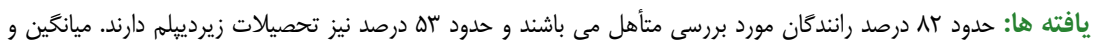

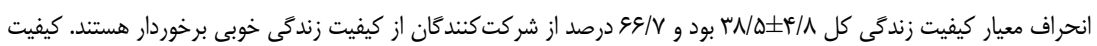

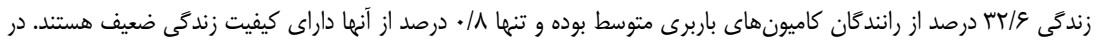

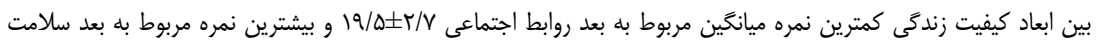

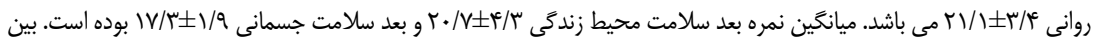

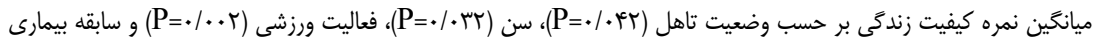

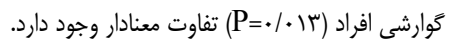

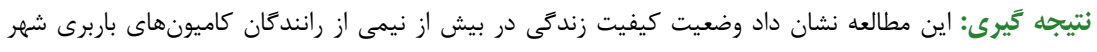

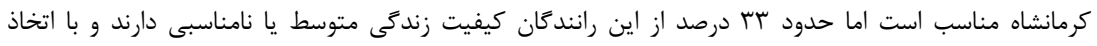

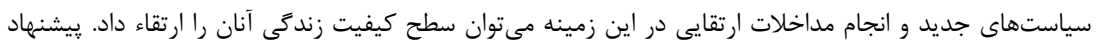

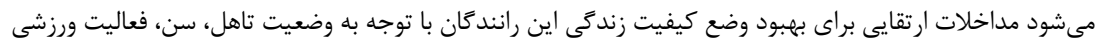

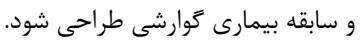
تعارض منافع: كزارش نشده /ست. منبع حمايت كنتلده: ندارد.

شيوه استناد به اين مقاله:

Mahnaz Solhi, Seyed Fahim Irandoost, Mitra Abolfathi. Investigating The Status of Quality of Life in Freight Truck Drivers of The City of Kermanshah and Its Influencing Factors. Iran Occupational Health. 2021 (01 Aug);18:15. 
شرايط جوى نامناسب، شيفتهاى كارى نامنظم، زياد

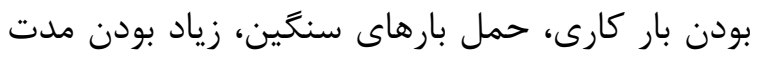

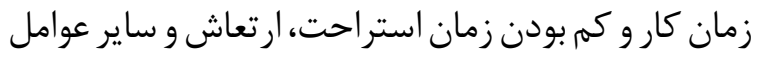

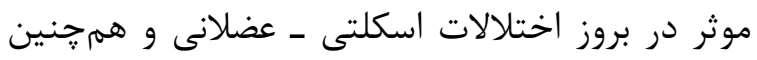

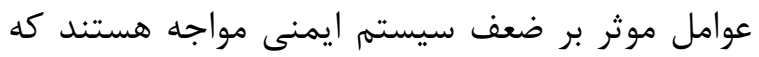

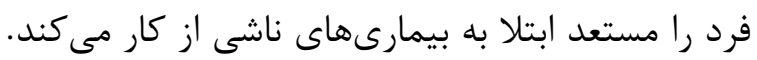

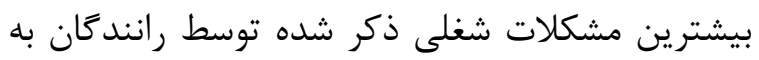

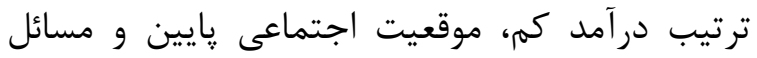

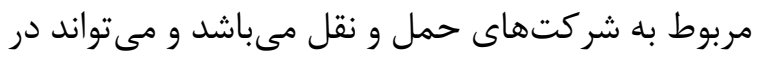

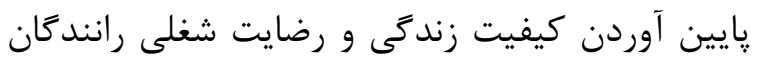

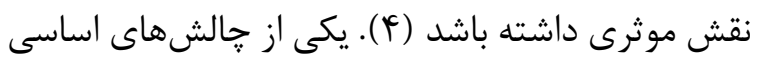

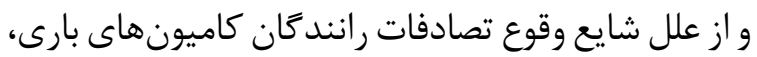

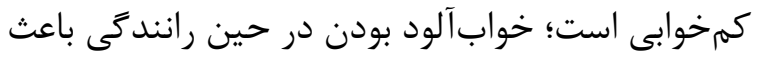

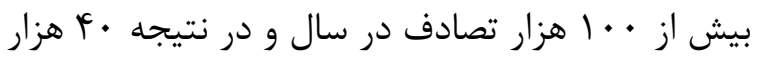

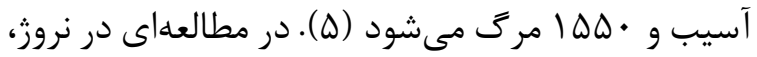

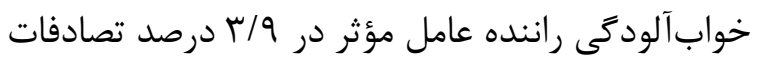

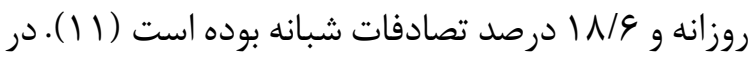

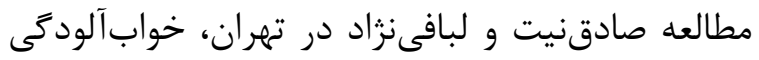

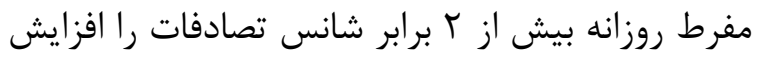

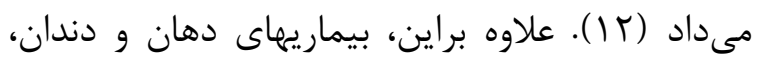

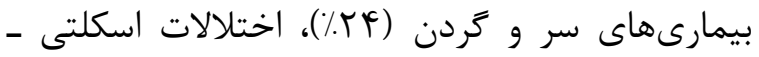

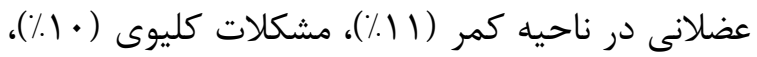

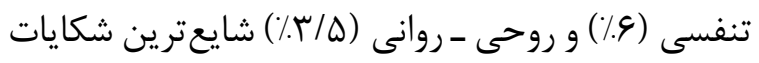

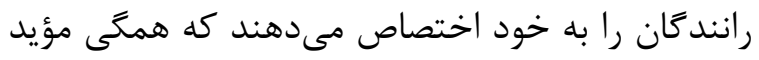

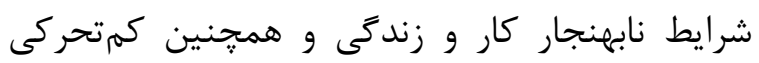

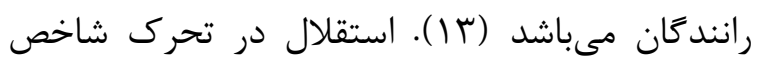

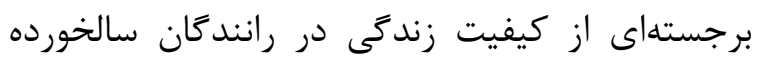

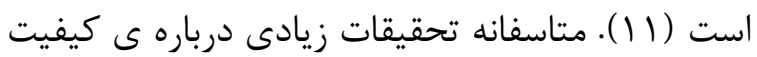

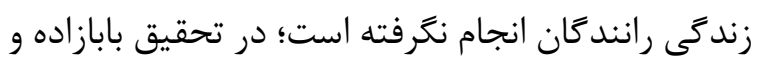

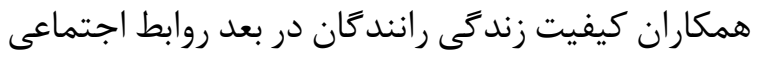

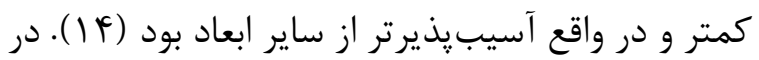

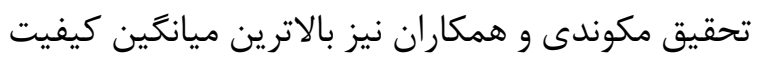

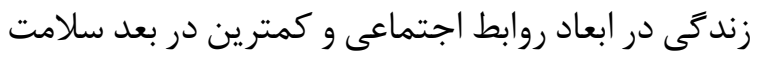

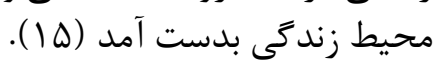

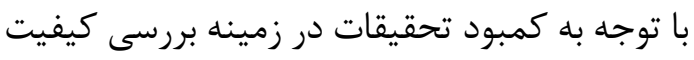

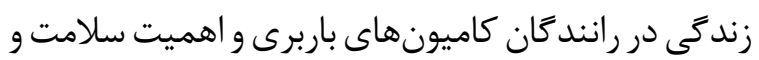

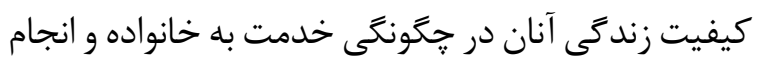

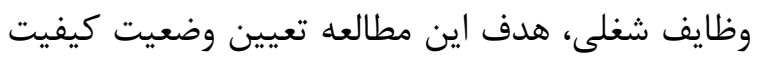

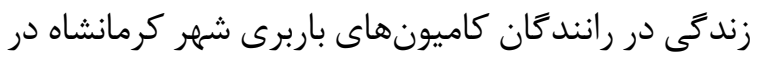

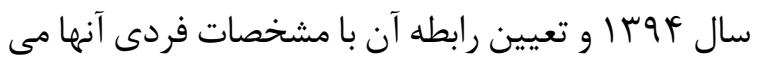

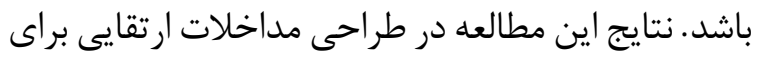

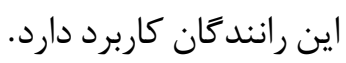

مقامله

امروزه حمل و نقل نقش معلش مهم و زيربنايى بر رشد

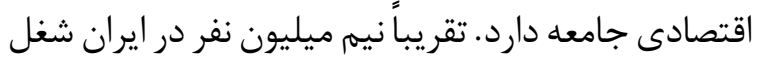

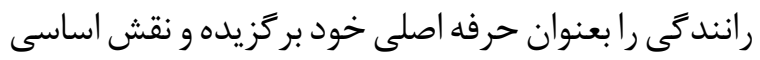

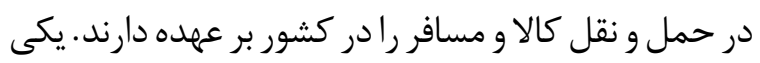

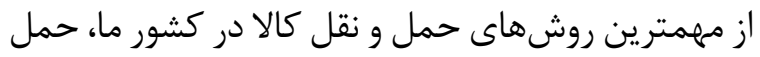

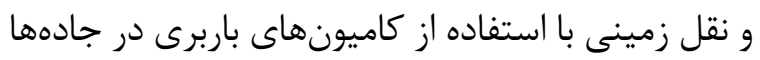

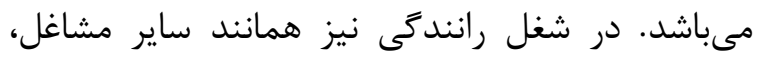

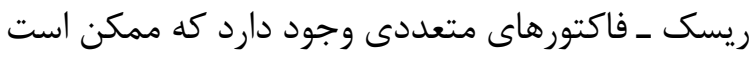

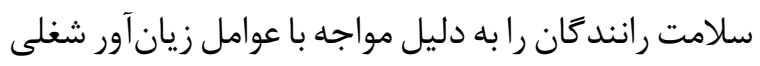

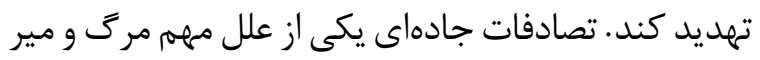

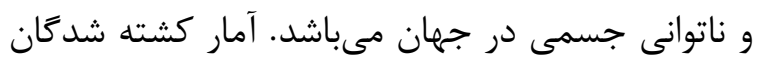

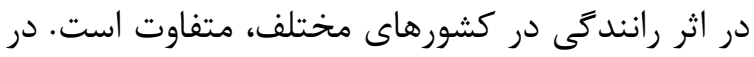

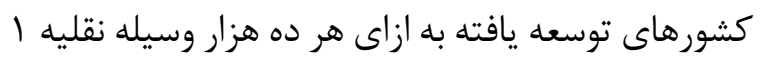

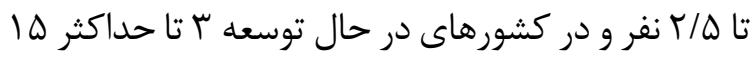

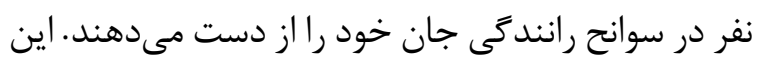

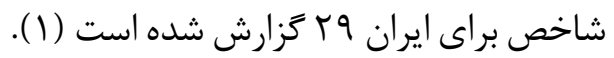

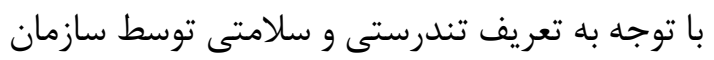

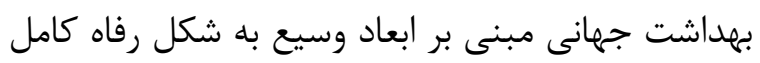

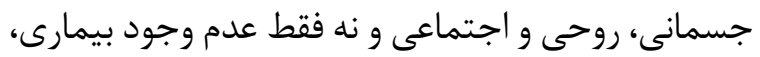

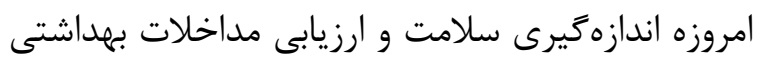

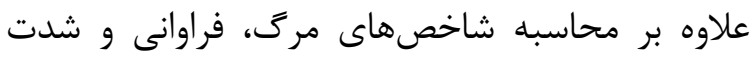

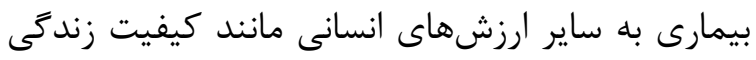

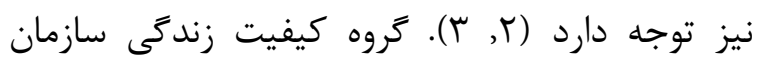

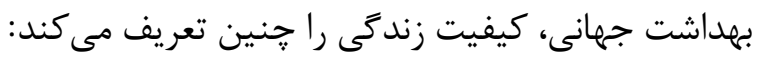

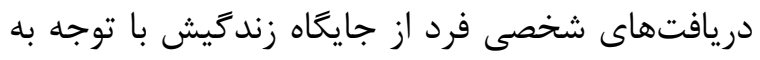

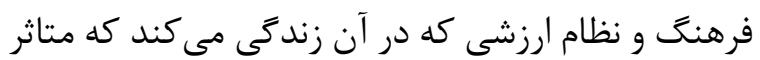

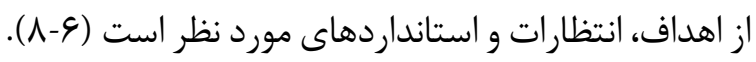

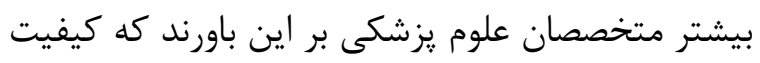

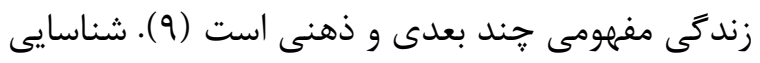

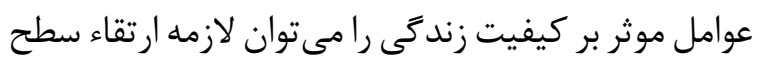

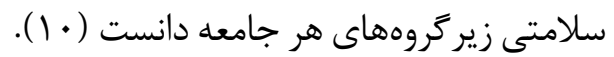

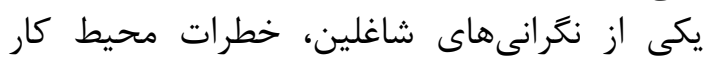

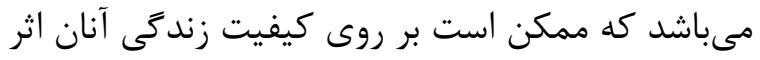

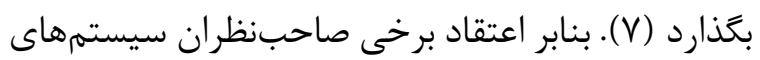

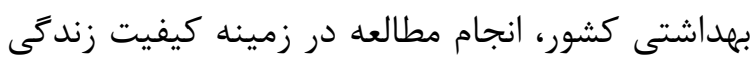

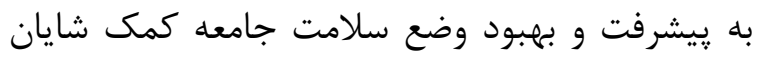

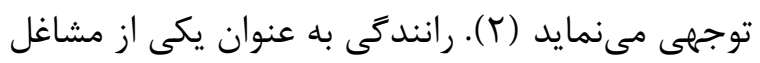

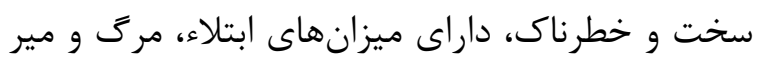

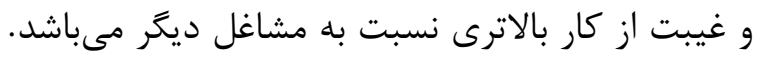

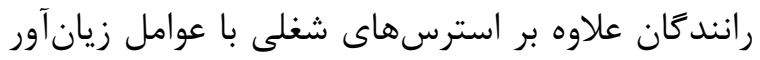

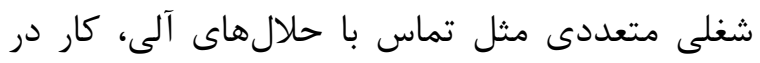


بافافتهها

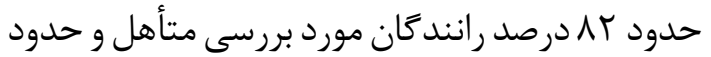

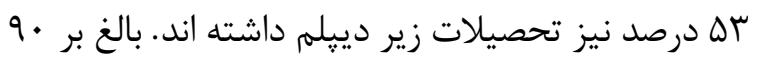

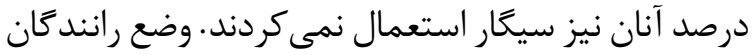

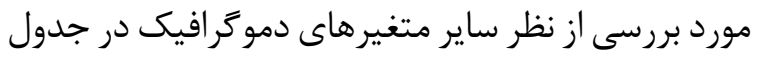

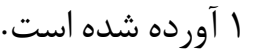

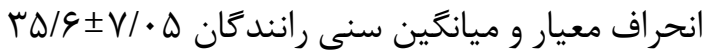

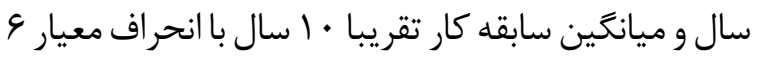

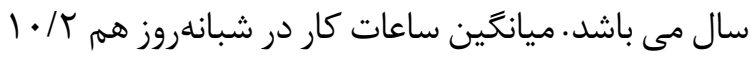

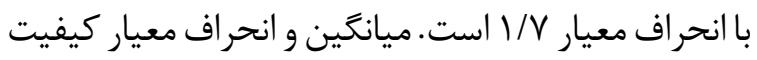

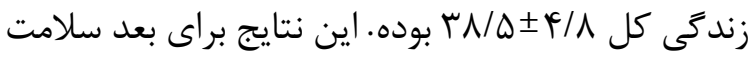

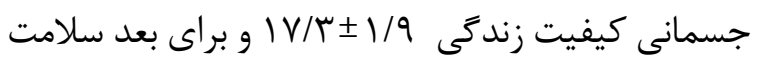
روانى

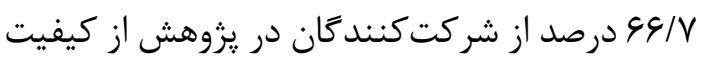

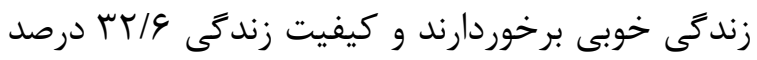

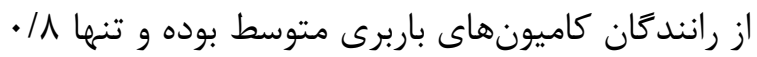

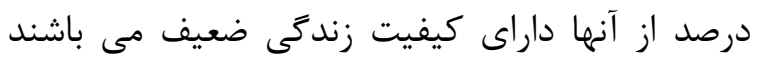

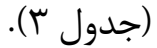

در بررسى دادهها، توزيع نمره كيفيت زند آنى، سلامت جسمانى و سلامت روانى داراى توزيع غير نرمال ندال است

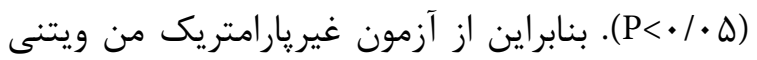

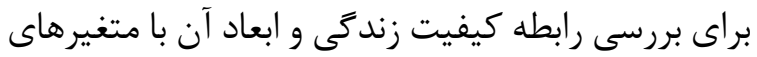

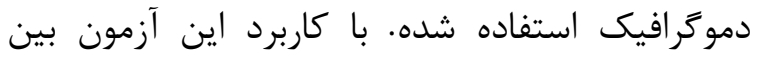

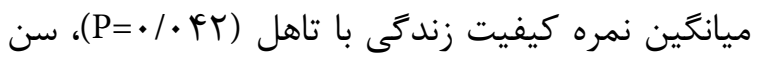

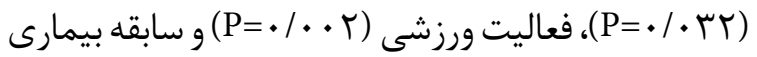

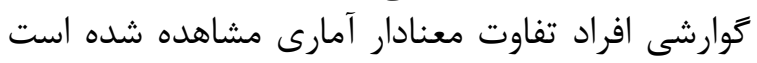

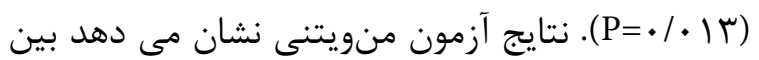

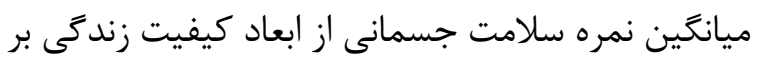

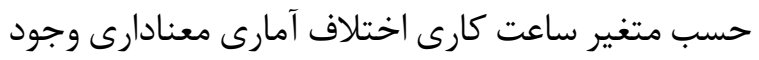

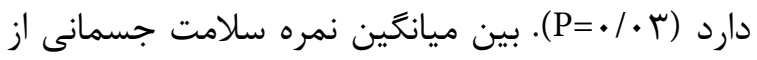

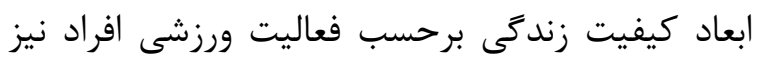

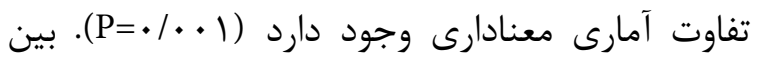

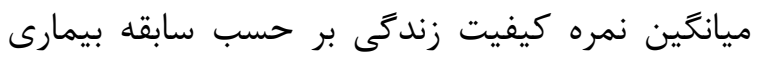

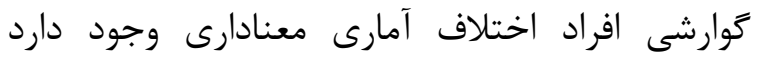

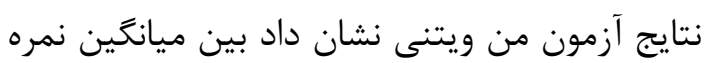

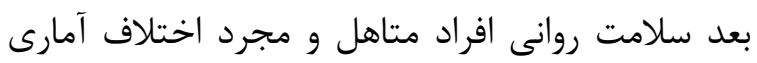

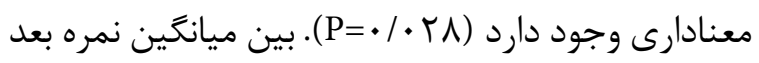

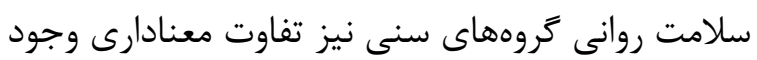

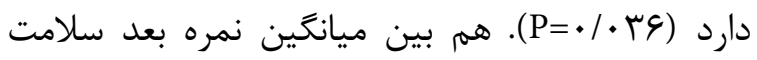

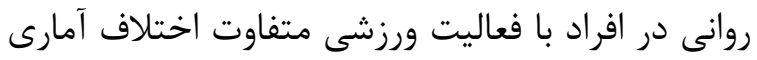

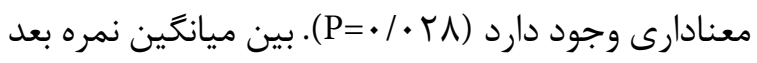

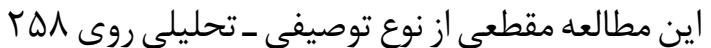

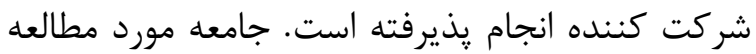

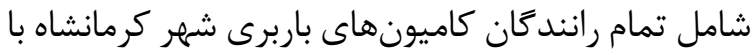

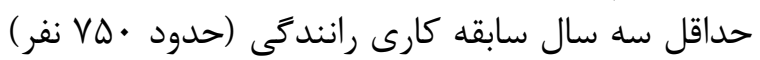

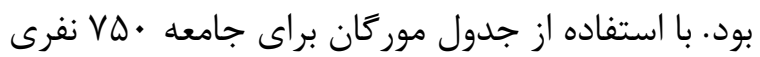

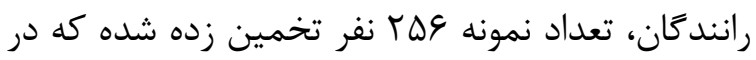

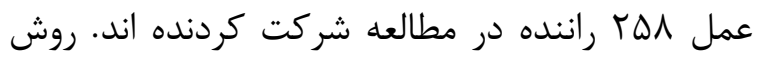

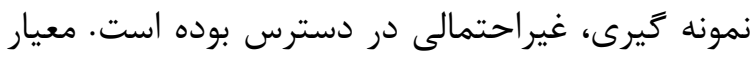

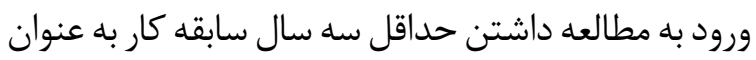
راننده كاميون باربرى در شهر كرمانشاه و رضايت آكاهانه

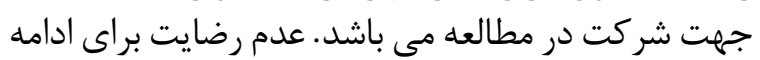

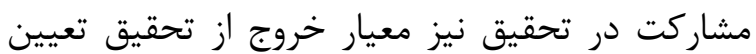

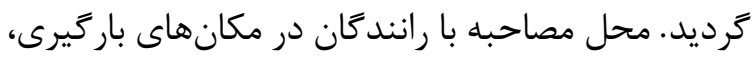

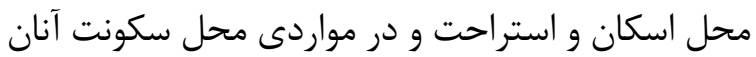
ابزار گردآورى اطلاعات برسشنامه استاندارد فرم كوتاه

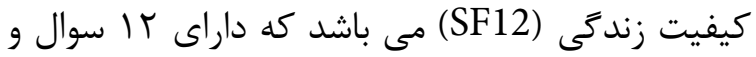

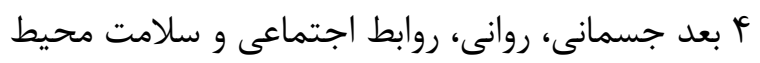

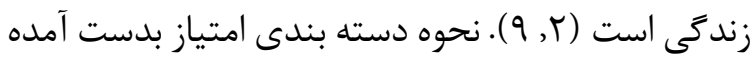

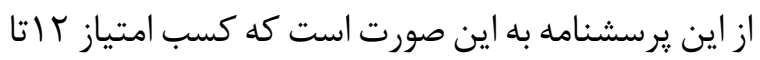

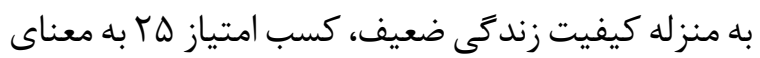

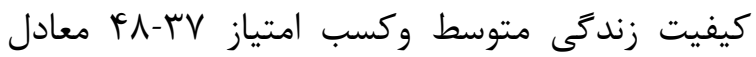

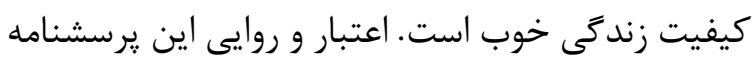

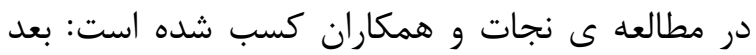

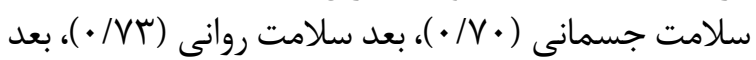

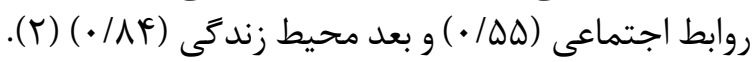

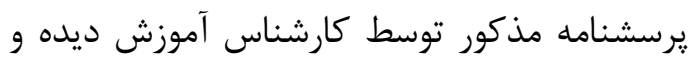

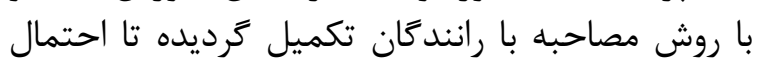

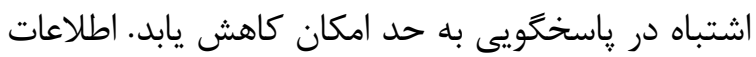

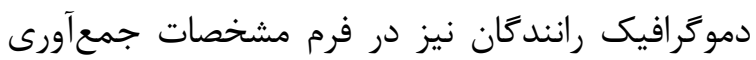

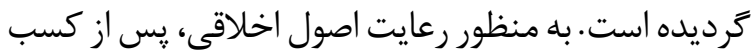

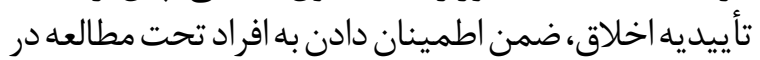

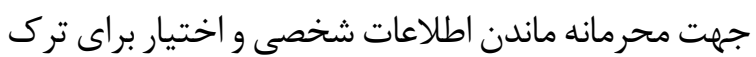

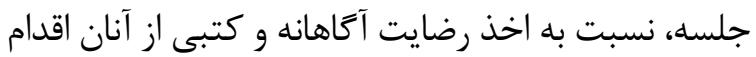

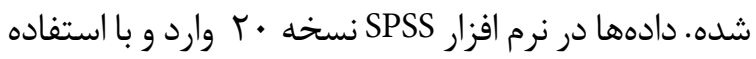

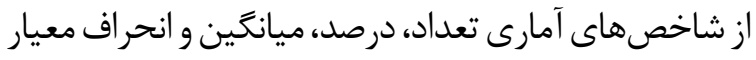

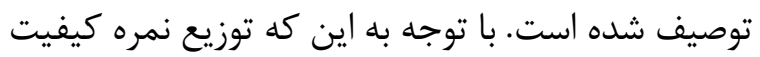

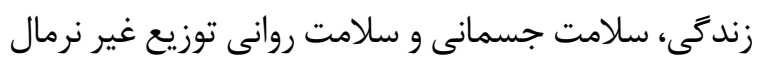

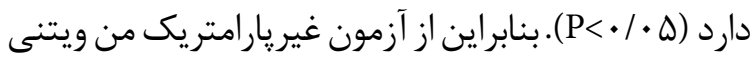

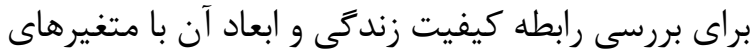
دموكر افيك استفاده شده راست. 
جدول ا. فراوانى نسبى مشخصات دموكرافيك رانندكان مورد مطالعه

\begin{tabular}{|c|c|c|c|}
\hline درصد & فراوانى & & \\
\hline $1 N / r$ & iv & مجرد & \multirow{2}{*}{ وضعيت تاهل } \\
\hline$\Lambda 1 / \Lambda$ & rII & متاهل & \\
\hline$\Delta T / V$ & Ire & زير دييلم & \multirow{3}{*}{ تحصيلات } \\
\hline$f 1 / 0$ & $1 \cdot v$ & دييلم & \\
\hline$\Delta / \Lambda$ & 10 & بالاتر از دييله & \\
\hline$r \mid / r$ & $\Delta \Delta$ & بلى بلى & \multirow{2}{*}{ عينك طبى } \\
\hline VA/V & $r \cdot r$ & خير & \\
\hline$r \cdot / r$ & $1 \cdot 4$ & بلى بلى & \multirow{2}{*}{ فعاليت ورزشى } \\
\hline$\Delta 9 / V$ & $1 Q F$ & خير & \\
\hline $9 / V$ & $r \Delta$ & دارد & \multirow{2}{*}{ مصرف سيكار } \\
\hline$q \cdot / r$ & 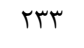 & 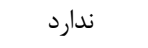 & \\
\hline$\cdot / \Lambda$ & r & 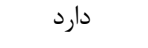 & \multirow{2}{*}{ استعمال مواد مخدر } \\
\hline $99 / r$ & rDG & 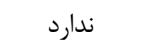 & \\
\hline$r / \Delta$ & 9 & 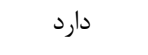 & \multirow{2}{*}{ سابقه بيمارى گَوارشى } \\
\hline $9 \& / 0$ & rFq & 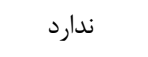 & \\
\hline$\cdot / 4$ & 1 & 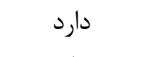 & \multirow{2}{*}{ سابقه بيمارى قلبى } \\
\hline $99 / 9$ & $r \Delta V$ & 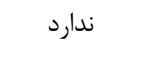 & \\
\hline $1 / 9$ & $r$ & دارد & \multirow{2}{*}{ سابقه يرفشارى خون } \\
\hline $91 / 4$ & TQF & 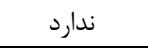 & \\
\hline
\end{tabular}

جدول r. ميانغين و انحراف معيار متغيرهاى كمى

\begin{tabular}{|c|c|c|c|c|}
\hline بيشترين & كمترين & انحر اف معيار & ميانگين & متغير \\
\hline \&A & tr & $f / \Lambda$ & rN/Q & نمره كلى كيفيت زندكى \\
\hline r. & 11 & $1 / 9$ & $\mid V / T$ & سلامت جسمانى \\
\hline rᄉ & 1. & $r / \mathcal{F}$ & $r M / l$ & سلامت روانى \\
\hline rq & it & $T / V$ & $19 / 0$ & روابط اجتماعى \\
\hline r. & 10 & $f / r$ & $r \cdot / V$ & سلامت محيط زندگى \\
\hline$\Delta 9$ & r & $V / \cdot \Delta$ & $r \Delta / \varphi$ & سن سن \\
\hline ra & 1 & $\Delta / 9$ & $9 / 9$ & سابقه كار \\
\hline М & r & $1 / V$ & $1 \cdot \pi$ & ساعت كار در شبانه روز \\
\hline
\end{tabular}

است (سب • • P=). همجنين با مقايسه ميانگين نمره بعد

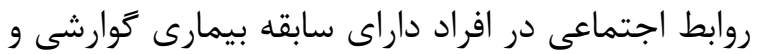

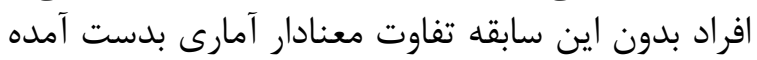

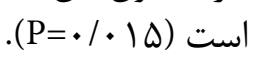
با كاربرد آزمون من ويتنى بين ميانگين نمره بعد بعد

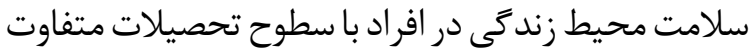

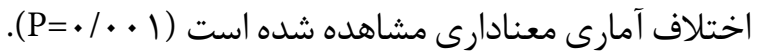

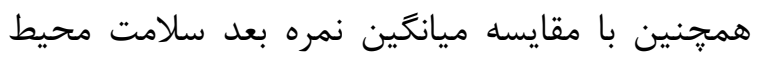

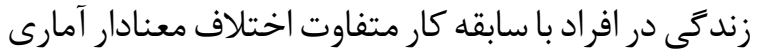

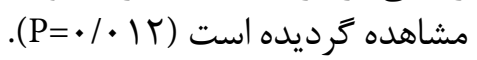

سلامت روانى در افراد با سابقه بيمارى گوارشى و بدون

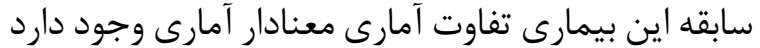
( $(\mathrm{P}=\cdot / \cdot \mathrm{FY})$ براساس آزمون من ويتنى مقايسه ميانگين نمره بعد

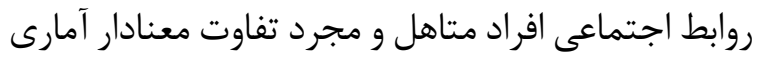

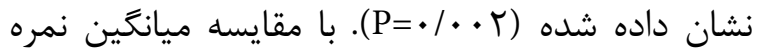
بعد روابط اجتماعى در افراد با تحصيلات مختلف تفاوت

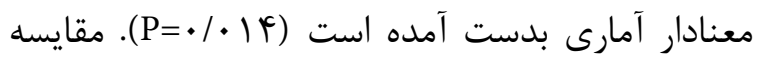
ميانگين نمره بعد روابط اجتماعى در افراد باد باد فعاليت

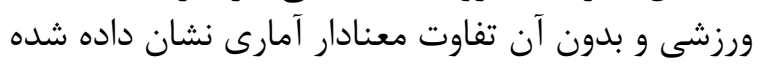


بررسى وضعيت كيفيت زندكى رانندكان كاميونهاى باربرى...

\begin{tabular}{|c|c|c|c|}
\hline \multicolumn{4}{|c|}{ جدول ب. نتايج توصيفى وضعيت كيفيت زندگى شركت كنندگان } \\
\hline درصد & 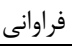 & طبقات متغير & 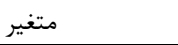 \\
\hline$\Leftrightarrow 9 / \mathrm{V}$ & IVT & 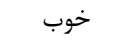 & $\|<$ \\
\hline rr/G & $\Lambda F$ & 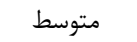 & ليعيد \\
\hline$\cdot / \Lambda$ & $r$ & ضعيف & v \\
\hline
\end{tabular}

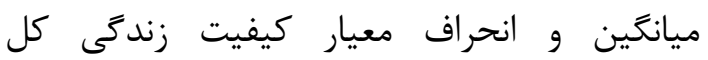

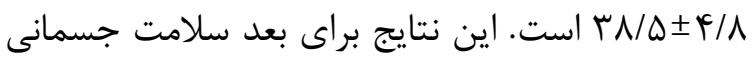

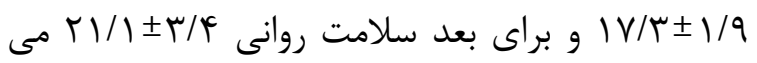

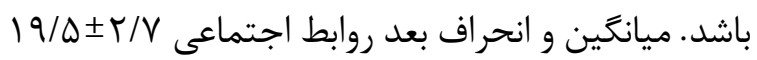

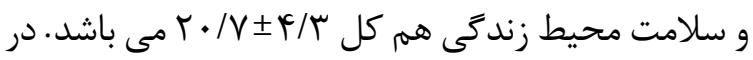

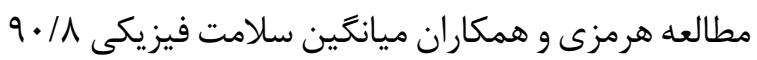

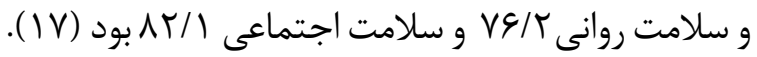

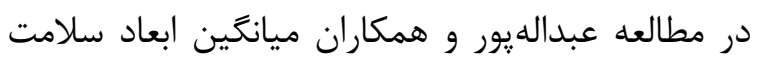

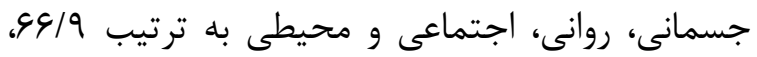

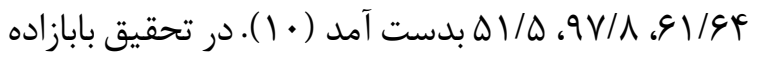

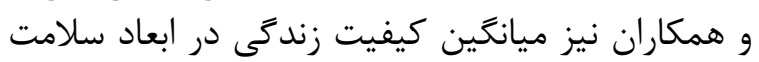

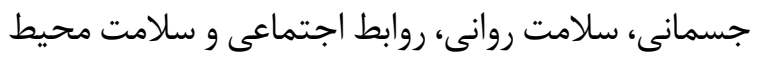

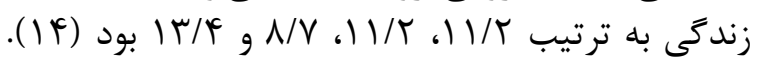

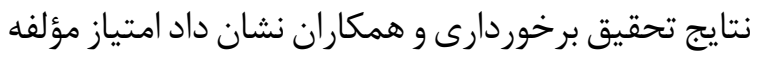

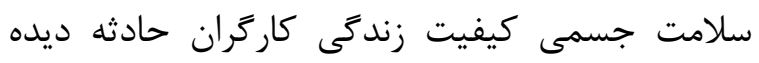

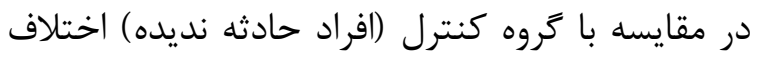

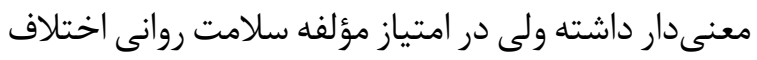

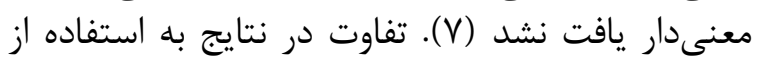

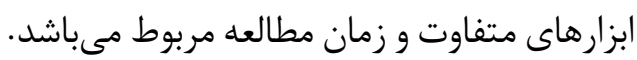

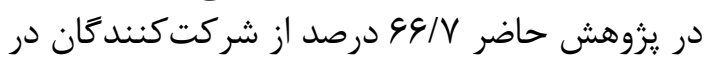

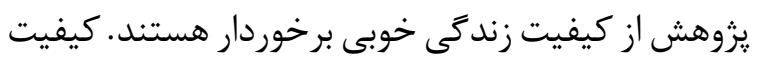
زندكى

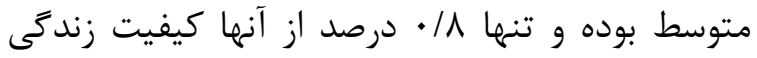

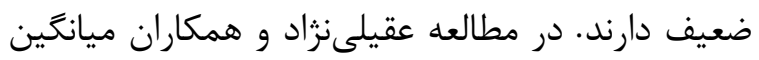

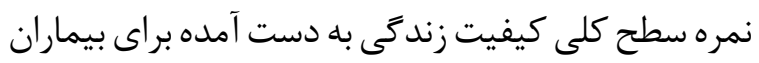

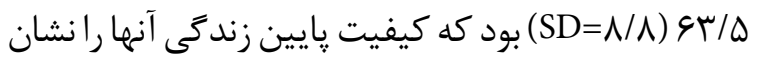

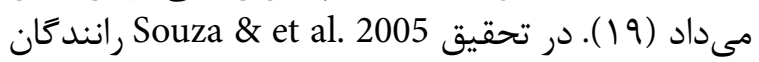

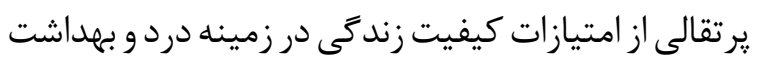

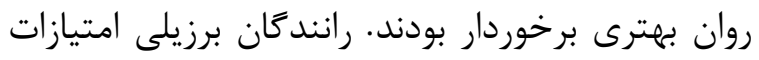

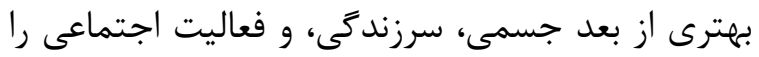

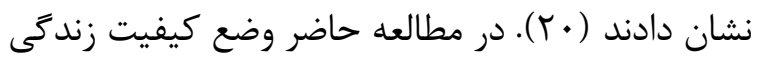

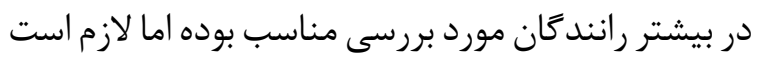

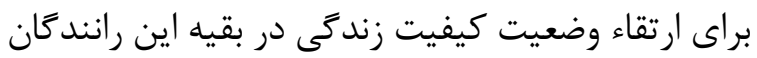

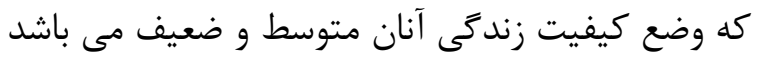
مداخلات ارتقايى برنامهريزى زندى شود. در اين مطالعه بين ميانگين نمره كيفيت زئند زندى كل

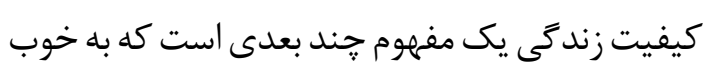

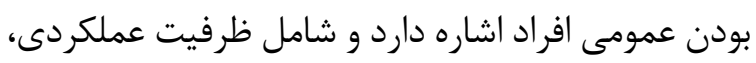

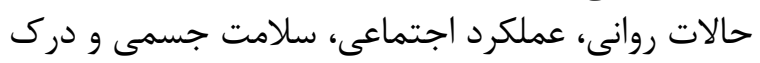

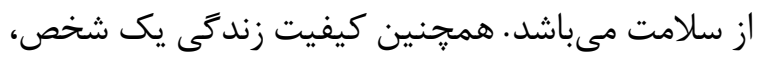

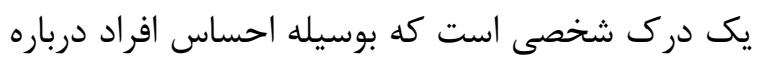

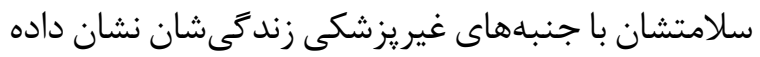

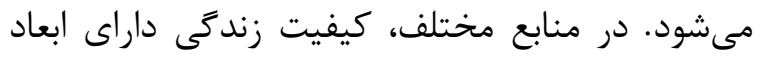

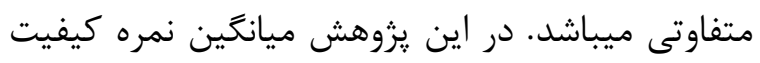

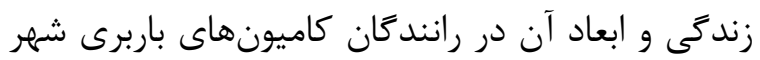

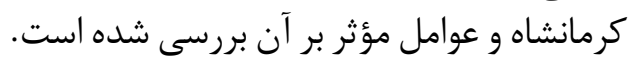

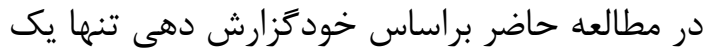

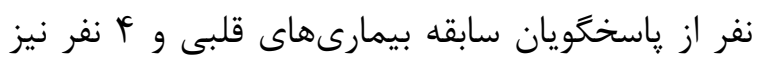

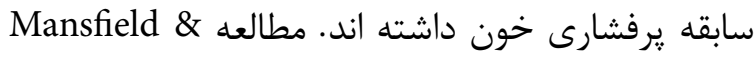

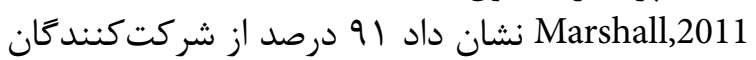

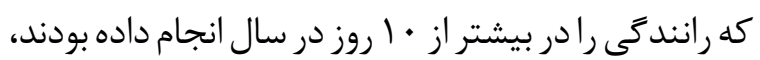

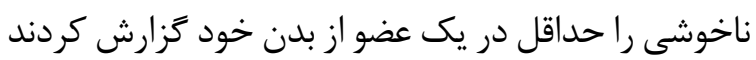

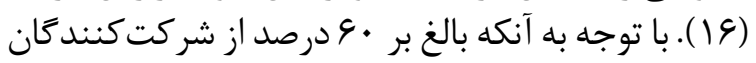

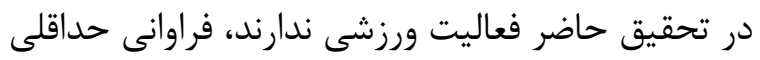

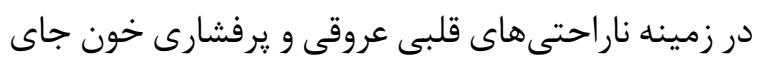

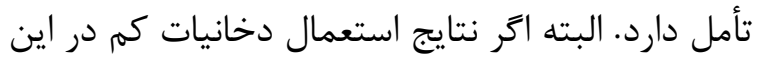

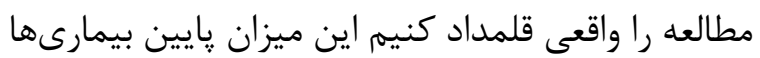
قابل توجيه است.

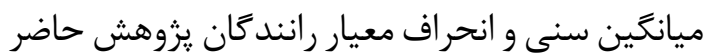

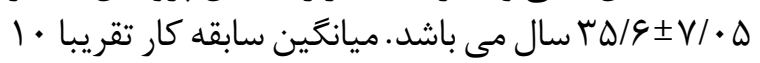

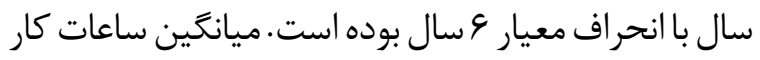

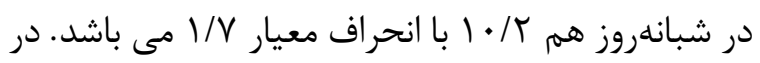

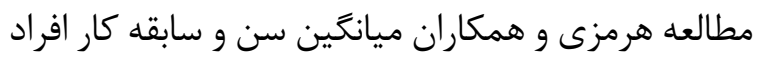

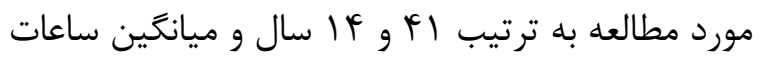

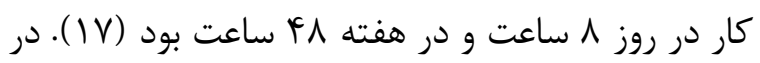

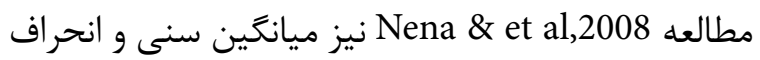

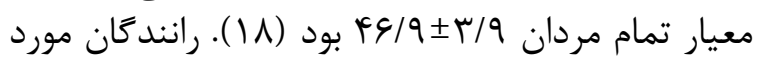

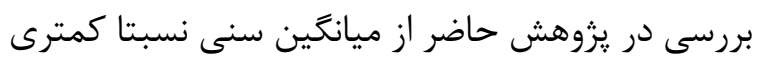

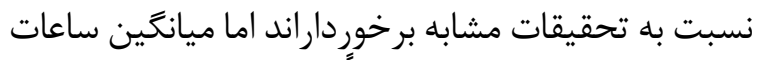

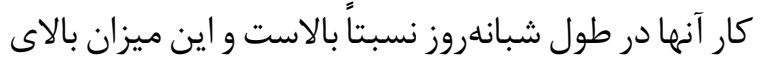
كار حتما بر كيفيت زندكى آنان آنان تأثير خواهد كذاشت Iran Occupational Health. 2021 (01 Aug);18: 15 
بيشتر و در رانندگان مسنتر، رانندكانى كه فعاليت

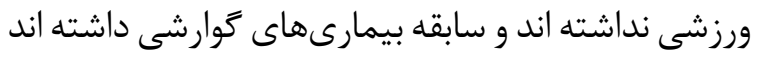

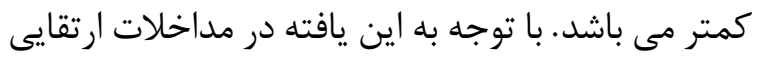

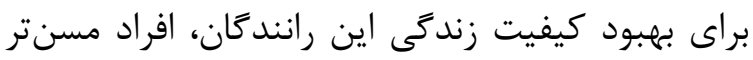

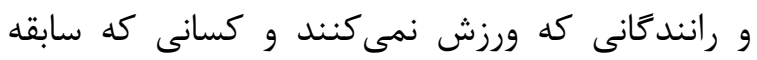

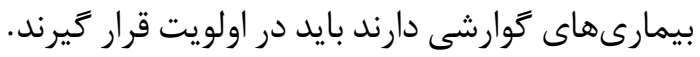

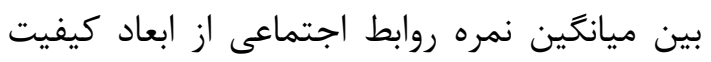

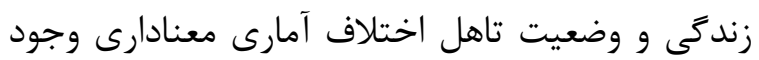

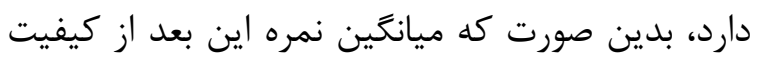

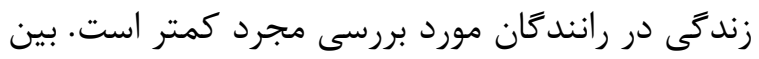

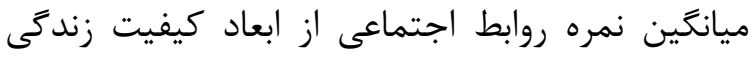

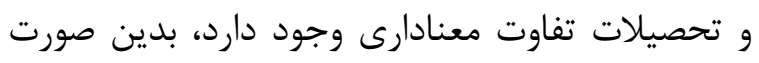

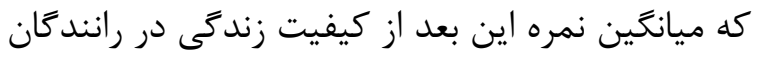

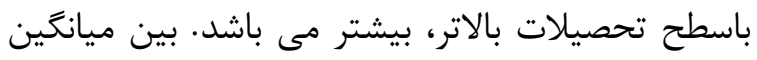

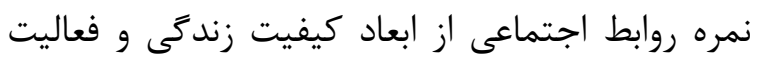

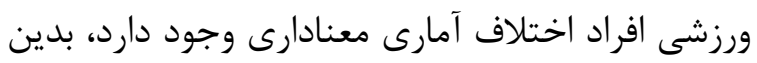

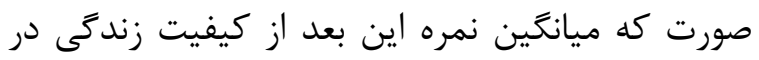

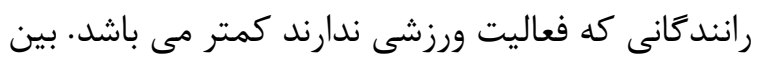

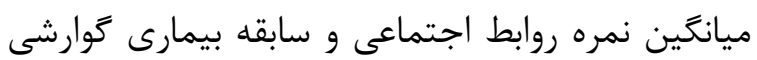

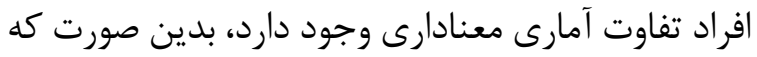

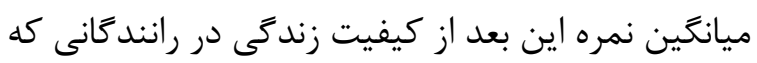

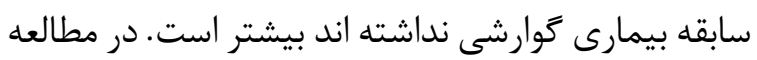

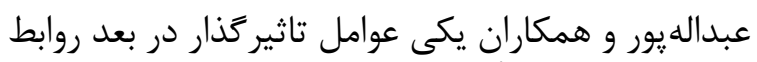

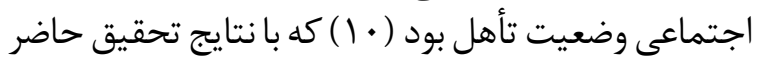

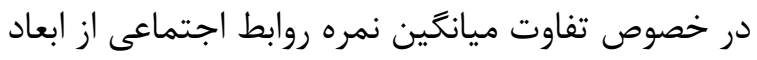

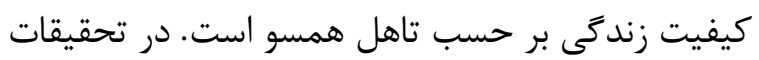

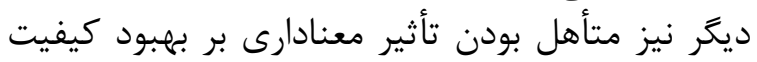

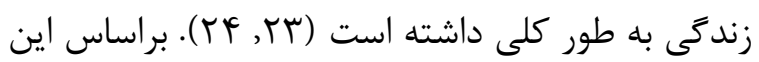

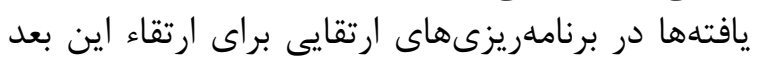

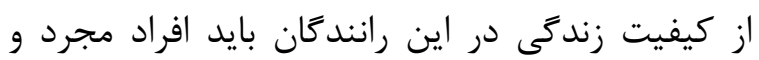

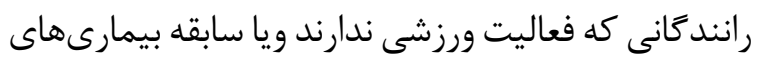

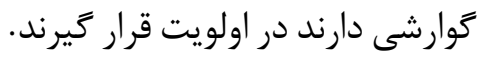

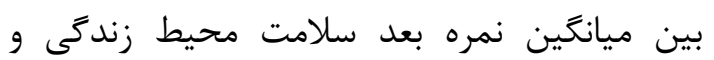

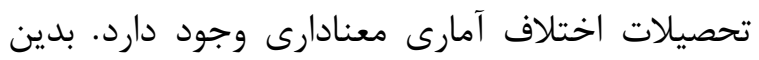

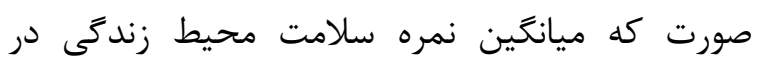

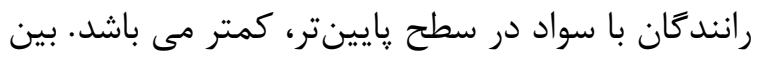

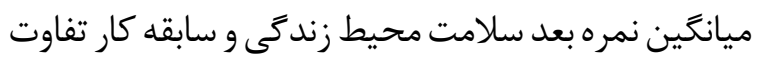

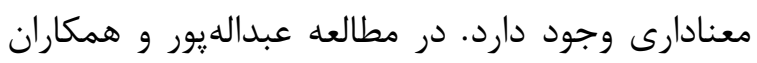

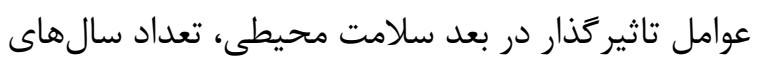

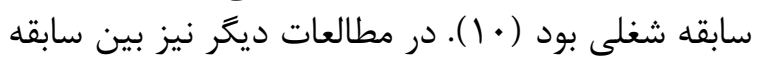

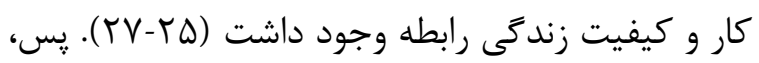

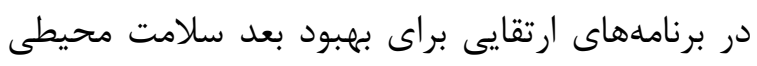

و وضعيت تاهل اختلاف آمارى معنادارى وجود داشته

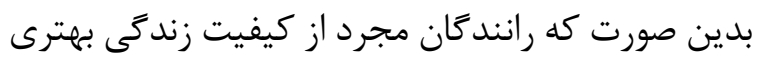

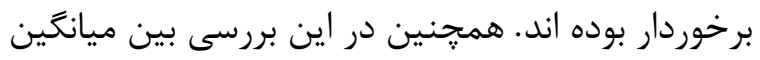
نمره كيفيت زند

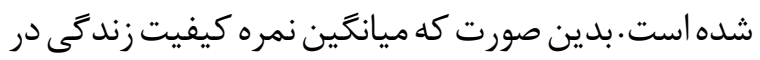

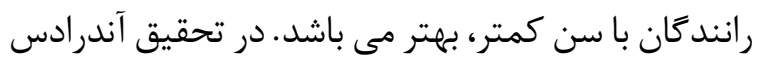

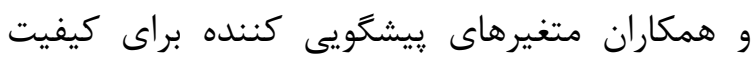

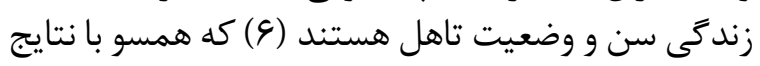

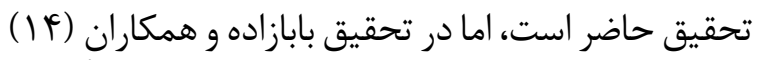

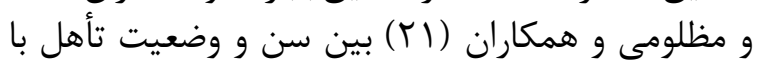

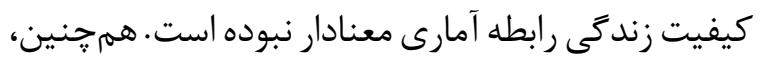

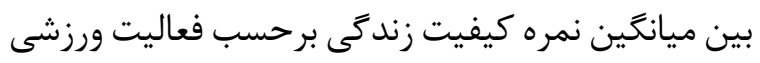

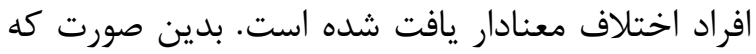

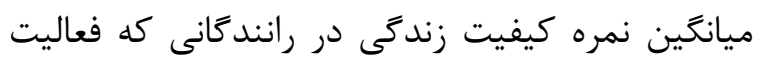

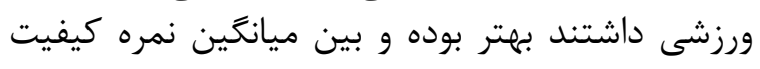

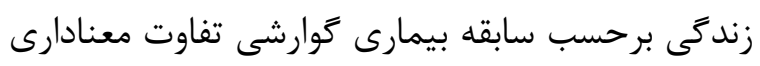

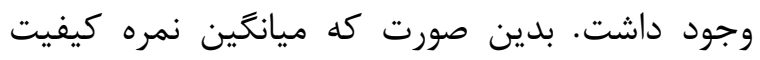

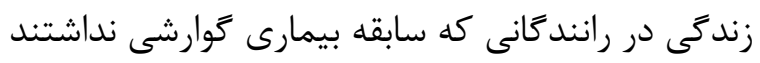

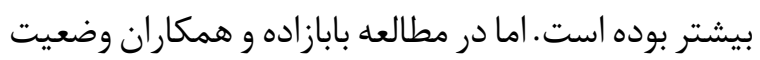

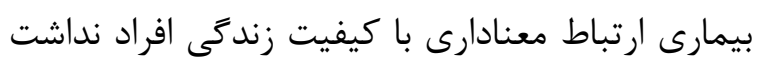

بين ميانخين نمره سلامت جسمانى از ابعاد كيفيت

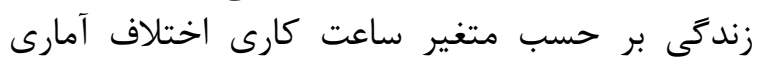

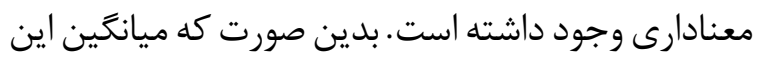

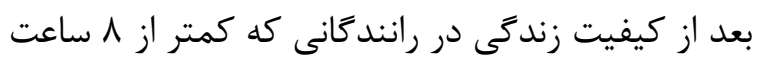

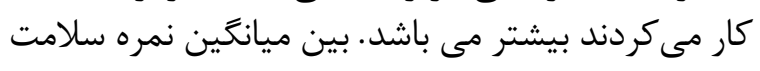

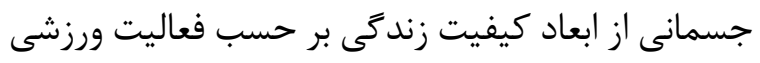

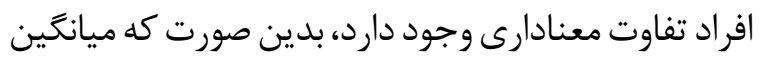

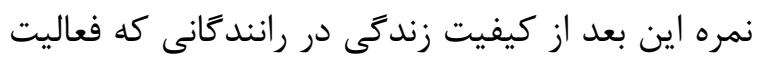

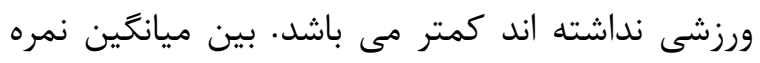

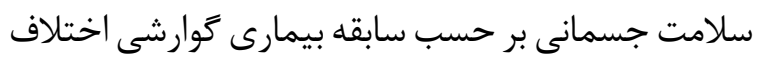

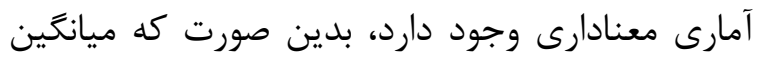

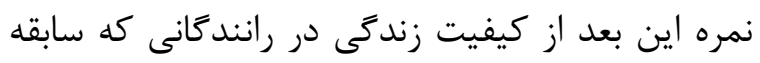

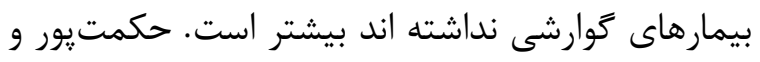

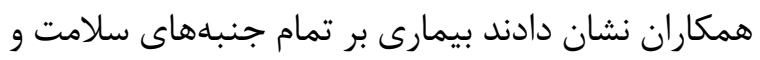

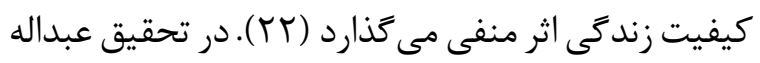

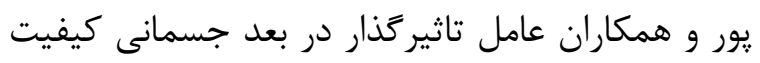

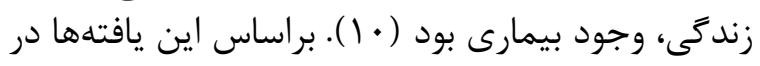

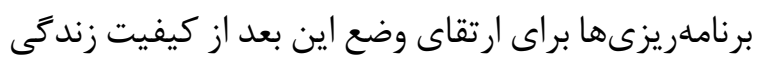

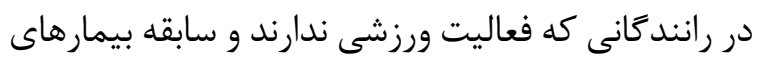

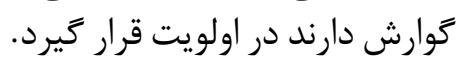

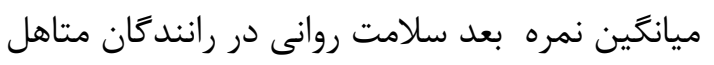


in RudbarLorestan Dam \& plant in 1389. Occupational Medicine Quarterly Journal. 2011;2(1):44-50.

8. Blay SL, Marchesoni MSM. Association among physical, psychiatric and socioeconomic conditions and WHOQOL-Bref scores. Cadernos de Saúde Pública. 2011;27:677-86.

9. Karimlou M, Zayeri F, Salehi M. Psychometric Properties of the Persian Version of the World Health Organization's Quality of Life Questionnaire (WHOQOL-100). Archives of Iranian Medicine (AIM). 2011;14(4):281-7.

10. Abdollahpour I, Salimi y, nedjat s, jorjoran shushtari z. Quality Of Life and Effective Factors on It among Governmental Staff in Boukan City. Urmia Medical Journal. 2011;22(1):40-7.

11. Williamson A, Lombardi DA, Folkard S, Stutts J, Courtney TK, Connor JL. The link between fatigue and safety. Accident Analysis \& Prevention. 2011;43(2):498515.

12. Sadeghniat K, Labafinezhad Y. Sleepiness among Iranian lorry drivers. Acta Medica Iranica 2007;45(2):149-52.

13. Saba A HM, Adamnejad S, Sayedalizadeh S. Health and safety of drivers in road transport and offer effective strategies to promote. Traffic management studies journal. 2010;15(4):103-11.

14. Babazadeh T, Taghdisi M, Sherizadeh Y, Mahmoodi $\mathrm{H}$, Ezzati E, Rezakhanimoghaddam $\mathrm{H}$, et al. The survey of health-related quality of life and its effective factors on the intercity bus drivers of the west terminal of Tehran in 2015. Community Health journal. 2017;9(1):19-27.

15. Makvandi M ZM. The survey of Quality of life and its domains in Ahvaz Islamic Azad university students Journal of Jontashapir 2012;2(4):191-200.

16. Mansfield N, Marshall J. Symptoms of musculoskeletal disorders in stage rally drivers and co-drivers. British journal of sports medicine. 2001;35(5):314-20.

17. Hormozi M. Study of the Quality of Life of Bus Drivers of Zahedan City. National Congress on Quality of Life and Health Promotion. 2011.

18. Nena E, Tsara V, Steiropoulos P, Constantinidis T, Katsarou Z, Christaki P, et al. Sleep-disordered breathing and quality of life of railway drivers in Greece. Chest. 2008;134(1):79-86.

19. Aghilinejad M LY, Golabadi M, Bajelan B. Life quality of workers' skin disorder caused by the Occupational Medicine Clinic Hospital Rasoul Akram in 1388. Journal of Medical Sciences, Iran's Army. 2010;7(4): 294-8.

20. Souza JC, Paiva T, Reimão R. Sleep habits, sleepiness and accidents among truck drivers. Arquivos de neuropsiquiatria. 2005;63(4):925-30.

21. Mazloumi A, Kazemi Z, Nasl-Saraji G, Barideh S.
كيفيت زندگى در اين رانندگان بايد افراد با تحصيلات

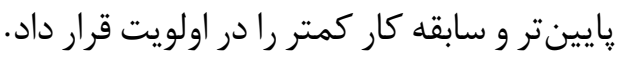

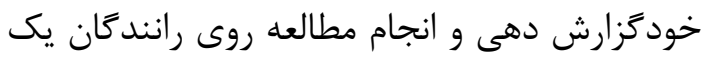

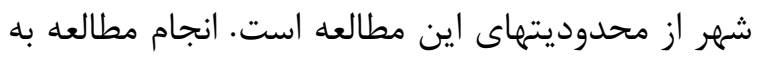
روش كيفى و تكنيك مصاحبه بر روى رانند

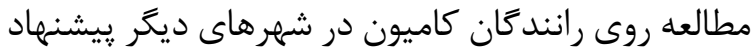
ميشود. نتيجه تيرى

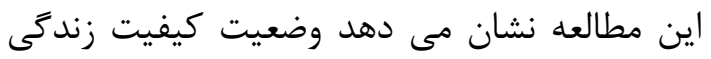

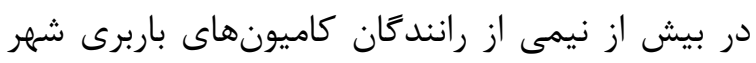

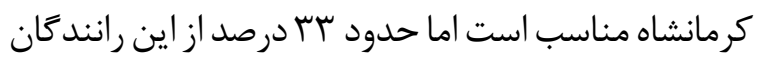

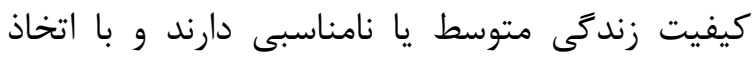
سياست هاى جديد و انجام مداخلات ارتقايى در اين زئن زمينه

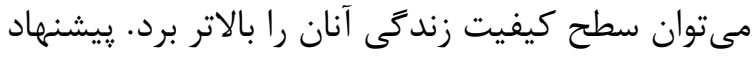

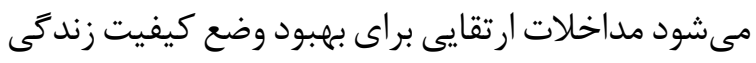

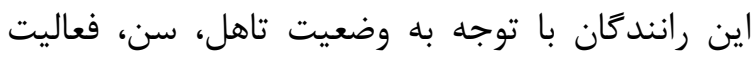
ورزشى و سابقه بيمارى گوارشى طراحى بـ شود.

منابع

1. Malek M, Halvani G, Fallah H. A study of the relationship between the Pittsburgh sleep quality index and road accidents among truck drivers. Occupational Medicine quarterly journal. 2011;3(1):14-20.

2. Nedjat S, Naieni KH, Mohammad K, Majdzadeh $\mathrm{R}$, Montazeri A. Quality of life among an Iranian general population sample using the World Health Organization's quality of life instrument (WHOQOLBREF). International journal of public health. 2011;56(1):55-61.

3. Abolfathi M, Pasdar Y, Kheiri M, Irandoost SF, Darabi F. The effect of consuming multivitamin/mineral supplements on elderly quality of life: Based on randomized control trial. Journal of Education and Health Promotion. 2021;10(1):63.

4. Vakili M, Eslami Farsani S, Hossein S, Dehghani Tafti M. Prevalence of depression and its related factors among truck drivers in Yazd Province-2008. Iran Occupational Health. 2010;6(4):69-76.

5. Safety O, Administration $H$. Guidelines for employers to reduce motor vehicle crashes. US Department of Labor, Washington, DC. 2015.

6. Andrades Barrientos L, Valenzuela Suazo S. Quality of life associated factors in Chileans hospitals nurses. Revista latino-americana de enfermagem. 2007;15(3):480-6.

7. BarkhordariA, ShiraziJ,HalvaniG, Baghianimoghaddam M, Fallahzadeh H, Sabzemakan L, et al. Review in relationship of quality of life with occupational accidents 
2012;22(93):112-22.

25. Khorsandi M, Jahani F, Rafiei M, Farazi A. Healthrelated quality of life in staff and hospital personnel of Arak University of Medical Sciences in 2009. Journal of Arak University of Medical Sciences. 2010;13(1):40-8.

26. Atoof F kA, Mehmandoost S and Sabery M. Quality of life and its related factors among nurses in Kashan Shahid- Beheshti hospital. Journal of Clinical Research in paramedical Science 2013;2(3):147-55.

27. Mokarami H, Taghavi S, Taban E. Psychosocial factors and Their Relationship to Health-Related Quality of Life in an industrial factory in Yasuj City. Iran Occupational Health. 2016;12(6):69-80.
Quality of working life assessment among train drivers in keshesh section of Iran Railway. International Journal of Occupational Hygiene. 2014;6(2):50-5.

22. Hekmatpour D JF, Behzadi F. . Study the quality of life among elderly women in Arak Arak Medical University Journal 2014;17(83):1-8.

23. Ghasemi SR, Rjabi Gilan N, Reshadat S. The survey of health-related quality of life in Kermanshah rural women and some related factors. J Mazand Univ Med Sci. 2014;24(109):186-94.

24. Navidian A. Quality of Life of Patients with Pulmonary Tuberculosis During Treatment Course. Journal of Mazandaran University of Medical Sciences. 